\title{
Timing offset calibration of CZTI instrument aboard ASTROSAT
}

\author{
A. Basu ${ }^{1}$, B. C. Joshi ${ }^{1}$, D. Bhattacharya ${ }^{2}$, A. R. Rao $^{3}$, A. Naidu ${ }^{1,4}$, M. A. Krishnakumar ${ }^{1,6}$, P. Arumugsamy ${ }^{1}$, \\ S. Vadawale ${ }^{5}$, P. K. Manoharan ${ }^{1,6}$, G. C. Dewangan ${ }^{2}$, A. Vibhute ${ }^{2}$, \\ N. P. S. Mithun ${ }^{5}$, and V. Sharma ${ }^{2}$

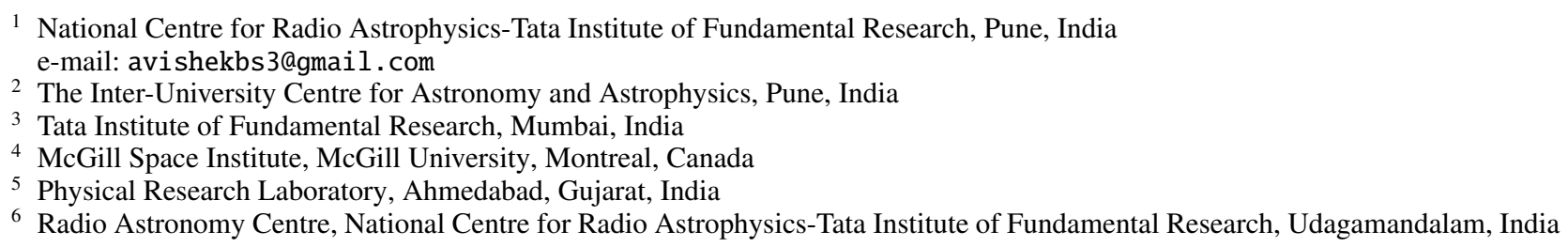 \\ Received 27 February 2018 / Accepted 28 May 2018
}

\begin{abstract}
Aim: Both the radio and the high-energy emission mechanism in pulsars is not yet properly understood. A multiwavelength study is likely to help better understand of such processes. ASTROSAT, the first Indian space-based observatory, has five instruments aboard that cover the electromagnetic spectrum from infra-red $(1300 \AA)$ to hard X-ray $(380 \mathrm{keV})$. The instrument relevant to our study is the Cadmium Zinc Telluride Imager (CZTI). CZTI is a hard X-ray telescope functional over an energy range of 20-380 keV. We aim to estimate the timing offset introduced in the data acquisition pipeline of the instrument, which will help in time alignment of high energy time-series with those from two other ground based observatories, viz. the Giant Meterwave Radio Telescope (GMRT) and the Ooty Radio Telescope (ORT).

Method: PSR B0531+21 is a well studied bright pulsar with closely aligned radio and hard X-ray pulse profiles. We used simultaneous observations of this pulsar with the ASTROSAT, the ORT, and the GMRT. As the pulsar resides in a very turbulent environment and shows significant timing noise, it was specially observed using the ORT with almost daily cadence to obtain good timing solutions. We also supplemented the ORT data with archival Fermi data for estimation of timing noise. We obtained the phase connected timing solution of the pulsar by estimating its dispersion measure variations and the timing noise. The timing offset of ASTROSAT instruments was estimated from fits to pulse arrival time data at the ASTROSAT and the radio observatories.

Results: We estimate the relative offset of ASTROSAT-CZTI with respect to GMRT to be $-4716 \pm 50 \mu s$. The corresponding offset with the ORT was $-29639 \pm 50 \mu s$ and Fermi-LAT was $-5368 \pm 56 \mu$ s respectively.
\end{abstract}

Key words. line: profiles - instrumentation: detectors - instrumentation: interferometers - ephemerides - reference systems pulsars: individual: B0531+21

\section{Introduction}

Simultaneous multiwavelength timing and spectral observations are very important in studying emission mechanisms of astrophysical objects with high energy emission, such as X-ray pulsars and X-ray binary systems (Yadigaroglu \& Romani 1995; Muslimov \& Harding 2003), to model their phase resolved spectra (Hu et al. 2017), and to constrain their magnetic field structure. Many of objects, particularly pulsars with known radio pulsations, require high-precision alignments of their radio and high energy light curves. Apart from constraining the nature of pair producing gaps, high precision alignment of their light curves can shed light on the nature of giant pulses $(\mathrm{GPs})^{1}$ in some of these pulsars (Joshi et al. 2004; Johnston et al. 2004; Johnston \& Romani 2004; Lundgren et al. 1995; Mikami et al. 2014; Hankins \& Eilek 2007).

Unfortunately, the behavior of electromagnetic radiation makes it impossible to observe all bands using a single instrument. In radio telescopes, the data are recorded in the form of

\footnotetext{
1 Intense nano second wide pulses, with typical intensities about 1000 times the mean pulse intensity, seen sporadically at radio frequencies in PSR B0531+21 and some other pulsars.
}

voltages as a function of time, whereas high energy detectors count the photons. The variability of both the radio and high energy emission necessitates having accurate synchronization of time, when the radiation at different frequency arrives at different observatories. This requires calibrating the delays in data acquisition and processing pipelines for each observatory through observations of sources with known time alignment. The calibration of fixed offsets for the instruments on board the first Indian multiwavelength space observatory, ASTROSAT, are presented in this paper for the first time.

ASTROSAT, launched in October 2015, has five instruments on board (Singh et al. 2014). These are the Cadmium Zinc Telluride Imager (CZTI; Bhalerao et al. 2017), Large Area X-ray Proportional Counter (LAXPC; Yadav et al. 2016), Soft X-ray Telescope (SXT; Singh et al. 2016), Ultra Violet Imaging Telescope (UVIT; Hutchings 2014), and Scanning Sky Monitor (SSM). This is a unique observatory providing multiwavelength coverage from $1300 \AA$ to $380 \mathrm{keV}$.

We have used Indian ground-based facilities such as the Giant Meterwave Radio Telescope (GMRT; Swarup et al. 1991) and the Ooty Radio Telescope (ORT; Swarup et al. 1971) simultaneously with the ASTROSAT to calibrate the fixed timing 


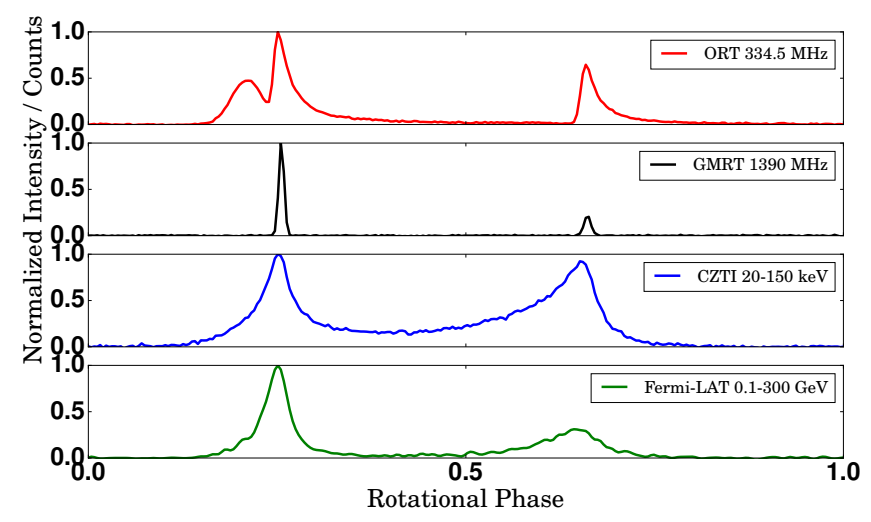

Fig. 1. Average pulse profile of PSR B0531+21 obtained using phase coherent average over all data with different instruments used in this study. The data were aligned using the offsets estimated in this study. The larger peak is called as main-pulse (MP), while the smaller peak is referred as inter-pulse (IP). The panels show the average profiles obtained with Fermi archival data, the CZTI, the GMRT and the ORT from bottom panel to top respectively. The radio profile were obtained with the GMRT at $1390 \mathrm{MHz}$ and with the ORT at $334.5 \mathrm{MHz}$.

offsets of various instruments. For this, we needed to use a standard calibrator with well known properties in all bands. We used the brightest high energy pulsar in the sky, the Crab pulsar (PSR B0531+21), for our calibration. PSR B0531+21 emits pulsed radiation from radio to very high energies (Abdo et al. 2010). The average light curve of the pulsar shows two peaks, with the larger peak at $1.4 \mathrm{GHz}$ defined as the main pulse (Fig. 1).

The pulsar's profile at low frequencies $(334.5 \mathrm{MHz}$ and $1.4 \mathrm{GHz}$ ) are aligned with the profile at optical and high energies. The main pulse at high energies leads the radio main pulse by $241 \pm 29 \mu s$ (>30 Mev; Kuiper et al. 2003), $344 \pm 40 \mu \mathrm{s}$ (2-30 keV; Rots et al. 2004) and $280 \pm 40 \mu \mathrm{s}$ (Kuiper et al. 2003). We used these reported intrinsic offsets to calibrate the instrumental offset of the instruments aboard the ASTROSAT.

This paper is organized as follows. The instruments aboard the ASTROSAT and the details of observations used for this study are described in Sect.2. The ephemeris for PSR B0531+21 were obtained using high cadence radio observations at $334.5 \mathrm{MHz}$ with the Ooty Radio Telescope (ORT). The analysis of these data and our calibration method is discussed in Sect. 3. We conclude with results and discussion in Sect. 4

\section{Observations}

The radio observations used the ORT and the GMRT, whereas the X-Ray observations were carried out using the CZTI instrument aboard ASTROSAT. We also used publicly available archival data from Fermi $^{2}$ mission. These instruments and the observational setup along-with the details of observations are described in this section.

\subsection{The Ooty Radio Telescope (ORT)}

The ORT is an offset parabolic cylindrical antenna of $530-\mathrm{m}$ length in north-south direction and 30-m width in east-west direction, sensitive to a single linear polarization, with system temperature of $150 \mathrm{~K}$ and the antenna gain of $3.3 \mathrm{~K} \mathrm{Jy}^{-1}$ (Swarup et al. 1971). PSR B0531+21 was observed as part of a

\footnotetext{
2 https://fermi.gsfc.nasa.gov/ssc/data/access/
}

larger pulsar-monitoring program (Krishnakumar et al., in prep.) since $2014 \mathrm{March}$ at $334.5 \mathrm{MHz}$ with a bandwidth of $16 \mathrm{MHz}$. The pulsar was observed daily for $15 \mathrm{~min}$ as part of this program and these observations were used to obtain monthly ephemeris of the pulsar as described below. The observations utilized the pulsar back-end at the ORT, called PONDER (Naidu et al. 2015), which employed real-time coherent dedispersion to obtain directly time-stamped average profiles of the pulsar using the monthly ephemeris generated by us. In PONDER, data acquisition is started at the rising edge of the minute pulse derived from global positioning system (GPS) and data are sampled in synchronization with observatory frequency standard, which was a Rubidium clock. The typical instrumental uncertainty on the time stamp was $200 \mathrm{~ns}$. Observations from 2015 September 01 (MJD 57226) to 2017 January 14 (MJD 57767) were used in this work.

\subsection{The Giant Meterwave Radio Telescope (GMRT)}

The GMRT is an interferometer consisting of thirty $45-\mathrm{m}$ fully steerable antennas (Swarup et al. 1971), 14 of which are arranged in a compact array within $1 \mathrm{~km}$ and the rest are distributed in three arms. We used the arm antennas nearest to the compact array along with the 14 compact array antennas to form a phased array at $1390 \mathrm{MHz}$, with an overall gain of $3.5 \mathrm{~K} \mathrm{Jy}^{-1}$. The two linear polarizations across $16 \mathrm{MHz}$ bandwidth from each antenna were digitized at Nyquist rate. The resultant time series was transformed with a 512 point fast Fourier transform (FFT) to obtain 256 channel voltages in the frequency domain. These were then compensated in the Fourier domain for instrumental phase for each antenna, determined by observing a point source $(3 \mathrm{C} 147)$ before each observations. The phase compensated voltages from all antenna in the phased array were then added and this coherent sum was recorded as 256 channel complex voltages, with a time-stamp for each block of 256 channels derived from observatory Rubidium frequency standard disciplined using one pulse per minute output of GPS. The recorded voltages were processed offline as described in Sect. 3. PSR B0531+21 was observed at the GMRT and the ORT simultaneously with ASTROSAT observations at four epochs. At other 13 epochs, the GMRT observations were not possible, so the ASTROSAT observations were carried out with the ORT only. The details of observations used are given in Table 1 .

\subsection{ASTROSAT-CZTI}

The Cadmium Zinc Telluride Imager (CZTI) instrument (Bhalerao et al. 2017) aboard ASTROSAT is a two-dimensional coded mask Imager with solid state pixelated Cadmium Zinc Telluride detectors of $976 \mathrm{~cm}^{2}$ total geometric area divided into four quadrants, each containing 4096 pixels. The instrument operates in the energy range $20-150 \mathrm{keV}$ for direct imaging, providing an angular resolution of $\sim 8$ arc-min within a field of view of $4.6^{\circ} \times 4.6^{\circ}$. Events recorded by the CZTI are time stamped with a resolution of $20 \mu \mathrm{s}$ as per the instrument clock. On ASTROSAT, the primary time standard is provided by a spacecraft positioning system (SPS) which generates a GPSsynchronized UTC reference. A synchronizing pulse is sent to all $\mathrm{X}$-ray payloads once in every 16 UTC seconds. The local clock values of all the instruments and of the SPS are recorded at each such pulse into a time correlation table (TCT). Events recorded in the CZTI are assigned UTC time-stamps by interpolation in the TCT. Accuracy of absolute time stamps thus assigned to 
Table 1. Brief summary of the observations.

\begin{tabular}{llll}
\hline \hline Telescope & BW or Energy range & Start MJD & Stop MJD \\
\hline ORT & $16 \mathrm{MHz}$ & 57226 & 57767 \\
GMRT legacy & $16 \mathrm{MHz}$ & 57316 & 57772 \\
ASTROSAT-CZTI & $20-150 \mathrm{keV}$ & 57303 & 57771 \\
Fermi-LAT & $0.1-300 \mathrm{GeV}$ & 57284 & 57800 \\
\hline
\end{tabular}

Notes. The participating telescopes and their payloads, bandwidth or the energy range employed for observations, and the range of MJD for which the data have been used are listed.

CZTI events are estimated to be within $\sim 3 \mu$ s standard deviations (Bhattacharya 2017). Unlike most other space observatories, the event time stamps in ASTROSAT are provided in the UTC system instead of TT. In order to derive the Barycentric arrival time of each event, these UTC time-stamps are processed, along with information regarding the orbital motion of ASTROSAT, through a modified version of the well-known AXBARY task of NASA HEASOFT package. The modification, made available under the name "as1bary", takes into account the additional bookkeeping required for leap seconds while processing UTC time stamps.

\section{Analysis}

\subsection{Analysis of radio data}

As mentioned in Sect.2.1, the data obtained at the ORT were already available as coherently dedispersed time-stamped profiles, which were used in the timing analysis described later. The GMRT spectral voltage data were coherently dedispersed offline using a pipeline developed by us. This pipeline first converts the spectral voltages to a voltage time series by taking an inverse FFT. The time-series is then convolved with a unity gain phase delay filter, representing the effect of inter-stellar medium as described in Naidu et al. (2015). Both the coherently dedispersed time series as well as an average profile, folded using the monthly ephemeris generated with the ORT data translated to the start time of the observations, were recorded for further analysis after integration to a resolution of $1 \mu \mathrm{s}$. The dispersion measure $^{3}(\mathrm{DM})$ used in the real-time coherent dedispersion carried out in PONDER back-end at the ORT used the DM value provided in the Jodrell Bank monthly ephemeris ${ }^{4}$ (Lyne et al. 1993) nearest to the epoch of observations. As the GMRT data were coherently dedispersed offline, subsequent to observations, DM derived from our timing analysis was used in this case to obtain the folded average profiles. The profiles, obtained with the ORT and the GMRT, were converted to PSRFITS ${ }^{5}$ format.

In addition to pulse profiles, the off-line analysis of the GMRT data also yielded data dedispersed to 64 sub-bands within the $16 \mathrm{MHz}$ band-pass. These were folded to 32 sub-integration for each of the $600 \mathrm{~s}$ observations at the GMRT and converted to PSRFITS.

First, a noise-free template was created from the observed average profiles for a given telescope as described below. The pulse at $334.5 \mathrm{MHz}$ is broadened due to multipath propagation in

\footnotetext{
3 Dispersion measure is the integrated column density of electrons in the line of sight of the pulsar, expressed in units of $\mathrm{pc} \mathrm{cm}^{-3}$.

4 http://www.jb.man.ac.uk/pulsar/crab.html

5 PSRFITS is an open data storage format, which is based on the flexible image transport system (FITS; Hotan et al. 2004).
}

the inter-stellar medium (Fig. 1). Furthermore, the pulse suffers a variable scatter-broadening at this frequency due to varying inhomogeneities in the Crab nebula. This can introduce a systematic error into the estimation of time-of-arrivals (TOAs) depending on the extent of scatter-broadening in the profiles used for forming the template. Hence, we chose a high signal-to-noise ratio $(\mathrm{S} / \mathrm{N})$ profile with minimum scatter broadening for creating a template at this frequency. As the scatter-broadening is negligible at $1390 \mathrm{MHz}$ (Fig. 1), the template at this frequency was obtained from a profile generated by aligning and averaging best average profiles from several epochs of observations. These profiles were then modeled as a sum of Gaussians, using tools in PSRCHIVE package (Hotan et al. 2004), to obtain a noise free templates. Separate templates were obtained for the ORT and the GMRT and these were aligned with the MP positioned at pulse phase 0.24 .

The average profile for each epoch at a given frequency were cross correlated with the noise free template for that frequency using a Fourier domain method (Taylor 1992) to obtain the shift at each epoch. The time-stamp at each epoch was adjusted by this shift to obtain TOA. These TOAs were used to refine the pulsar rotation parameter using pulsar timing package TEMPO2 (Hobbs et al. 2006) ${ }^{6}$. In brief, this technique (called pulsar timing) compares the observed TOAs with those predicted by an assumed rotation model of the pulsar, keeping track of every rotation and minimizing the timing residuals through a least square fit PSR B0531+21 shows rotational irregularities in the form of timing noise (Scott et al. 2003) and glitches (Wang et al. 2012). These irregularities can significantly affect the residuals leading to phase ambiguities. Thus, closely spaced observations of pulsar are required to keep track of pulse phase and maintain phase connection. Our experiment used the ORT for high cadence observations of the pulsar to achieve the required phase connection.

The TOAs from the ORT data were divided in 30 day intervals and local fits to the spin frequency (F0) and its derivatives (F1 and F2) were performed at an epoch in the center of each 30-day interval. These 30-day ephemerides were then used for folding the high energy data as well as the $1390 \mathrm{MHz}$ GMRT data. The details of full phase connected timing analysis are described in Sect. 3.3.

\subsection{Analysis of high energy data}

\subsubsection{Analysis of Fermi-LAT data}

We used the available archival data from Fermi-LAT ${ }^{7}$ and extracted all events in a 3 -deg radius around the position of PSR

\footnotetext{
6 http://www.atnf.csiro.au/research/pulsar/tempo2/

7 https://fermi.gsfc.nasa.gov/cgi-bin/ssc/LAT/ LATDataQuery.cgi
} 


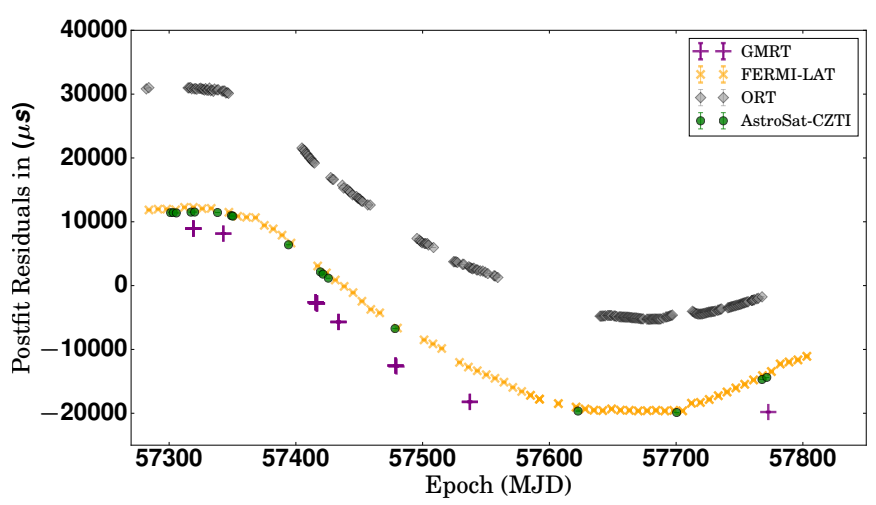

Fig. 2. Phase connected TOAs from the Fermi-LAT (yellow cross markers), the ASTROSAT CZTI (green circles), the GMRT (purple plus markers) and the ORT (gray diamonds) observations. The phase connection was obtained with the high cadence TOAs derived from the ORT observations and then applied to TOAs from other telescopes. The systematic pattern in the timing residuals is due to timing noise. The TOAs for different telescopes are offset with each other due to relative delays in the data acquisition at each telescope.

B0531+21 in the energy range of $0.1-300 \mathrm{GeV}$. These were then split into separate event files, each spanning seven days using Fermi science tools ${ }^{8}$. The event times were referenced to the solar system barycenter (SSB) and the events were folded using the Fermi plugin (Ray et al. 2011) of TEMPO2 with the ephemeris obtained in Sect.3.1. A template for the averaged light curve in gamma-ray energies was constructed in a manner similar to the radio data and was aligned with the $1390 \mathrm{MHz}$ and the $334.5 \mathrm{MHz}$ templates. The TOAs for each seven-day integrations were then derived by cross-correlating with this template and used in the subsequent timing analysis.

\subsubsection{Analysis of ASTROSAT data}

As mentioned before, instruments on board ASTROSAT provide individual photons with time-stamps derived from a satellite positioning system (SPS). The time tags of the photon were converted to solar system barycentre using the position of satellite in a code called aslbary. The barycentered events were then binned across 256 pulse phase bins using the ephemeris obtained in Sect. 3.1. The binned profile were then written as PSRFITS files.

CZTI instrument has four quadrant detectors. Hence for a good $\mathrm{S} / \mathrm{N}$, we needed to combine data from all the quadrants. We checked the alignment of the individual detector data by folding the photons from each quadrant separately as well as after combining the data from all quadrants. The profiles, so obtained, are shown in Fig. 3, where the phases were appropriately aligned using TEMPO2. All the analysis in this paper uses the data combined from all four quadrants.

Separate profile templates were constructed for CZTI data in a manner similar to the radio template. These were aligned with the the Fermi-LAT, the GMRT and the ORT templates. Finally, the CZTI template was cross-correlated with the observed profiles for CZTI to obtain TOAs in a manner similar to Fermi data. These were subsequently used in the timing analysis described in the next section.

Timing offsets evaluated from observed differences in the Crab pulsar phase may suffer from ambiguities amounting to in-

\footnotetext{
8 https://fermi.gsfc.nasa.gov/ssc/data/analysis/ scitools/overview.html
}

Table 2. Reference timing solution for PSR B0531+21 after accounting for the timing noise and DM variations using the multiband observations presented in this paper.

\begin{tabular}{|c|c|}
\hline Pulsar parameter & Value \\
\hline RAJ (hh:mm:ss) & $05: 34: 31.973$ \\
\hline DECJ (dd:mm:ss) & $+22: 00: 52.06$ \\
\hline $\mathrm{F} 0(\mathrm{~Hz})$ & $29.6607409(4) \mathrm{E}-7$ \\
\hline $\mathrm{F} 1\left(\mathrm{~Hz} \mathrm{~s}^{-1}\right)$ & $-3.6937842(9) \mathrm{E}-10$ \\
\hline $\mathrm{F} 2\left(\mathrm{~Hz} \mathrm{~s}^{-2}\right)$ & $1.1905(3) \mathrm{E}-20$ \\
\hline PEPOCH (MJD) & 57311.000000136 \\
\hline POSEPOCH (MJD) & 40675 \\
\hline DMEPOCH (MJD) & 57311.000000136 \\
\hline $\mathrm{DM}\left(\mathrm{pc} \mathrm{cm}^{-3}\right)$ & 56.7957 \\
\hline PMRA (mas year ${ }^{-1}$ ) & -14.7 \\
\hline PMDEC (mas year $\left.{ }^{-1}\right)$ & 2 \\
\hline WAVE_OM $\left(\right.$ year $\left.^{-1}\right)$ & 0.0054325986245627 \\
\hline WAVEEPOCH (MJD) & 57311.000000136 \\
\hline DMMODEL $\left(\mathrm{pc} \mathrm{cm}^{-3}\right)$ & 56.7957 \\
\hline
\end{tabular}

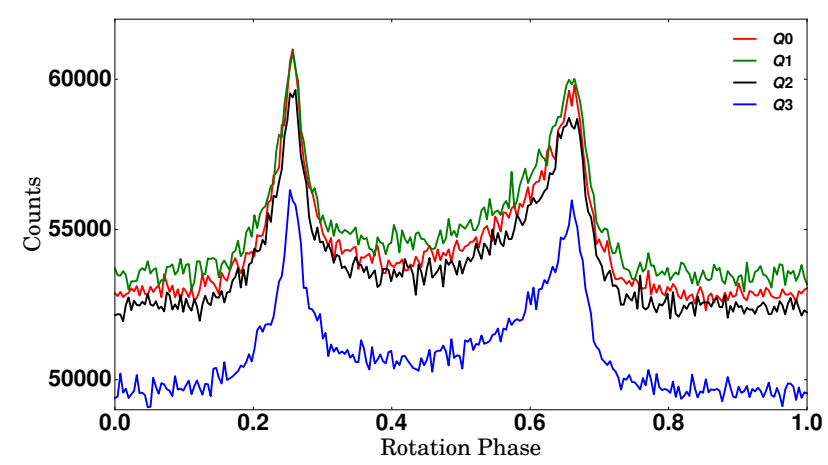

Fig. 3. Phase aligned profiles of PSR B $0531+21$ using four different detectors of CZTI arranged in a four quadrant fashion (Q0, Q1, Q2 and Q3).

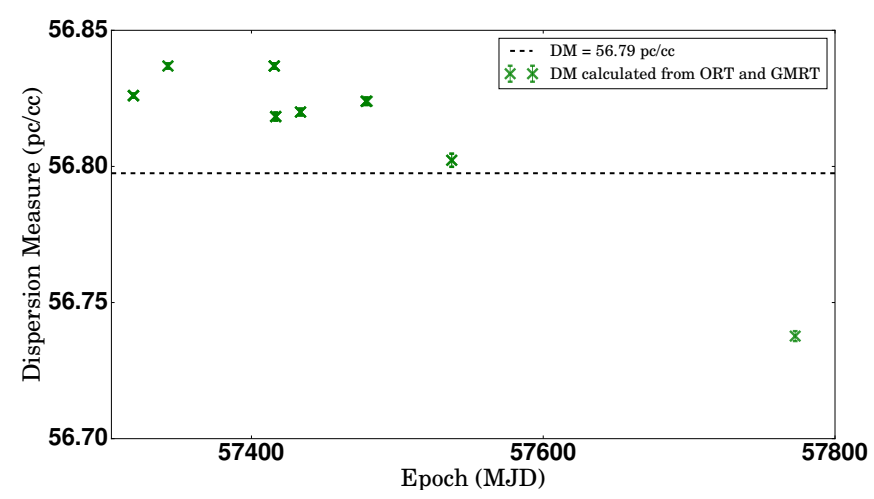

Fig. 4. Dispersion measure (DM) variations with observations epoch.

tegral multiples of the pulse period. To test whether the offset between AstroSat-CZTI and Fermi could be as large or larger than 33 milliseconds, we compared the detection times of gamma ray bursts by these two missions. In particular, the bright, short burst GRB170127C (Bissaldi et al. 2017; Sharma et al. 2017) provided the best $\mathrm{S} / \mathrm{N}$ for this test. We binned the UTC light curves from Fermi-GBM and AstroSat-CZTI at $10 \mathrm{~ms}$ resolution. The cross-correlation function of these two light curves showed a sharp peak at a delay of $0.0 \mathrm{~ms}$ with a formal error of 


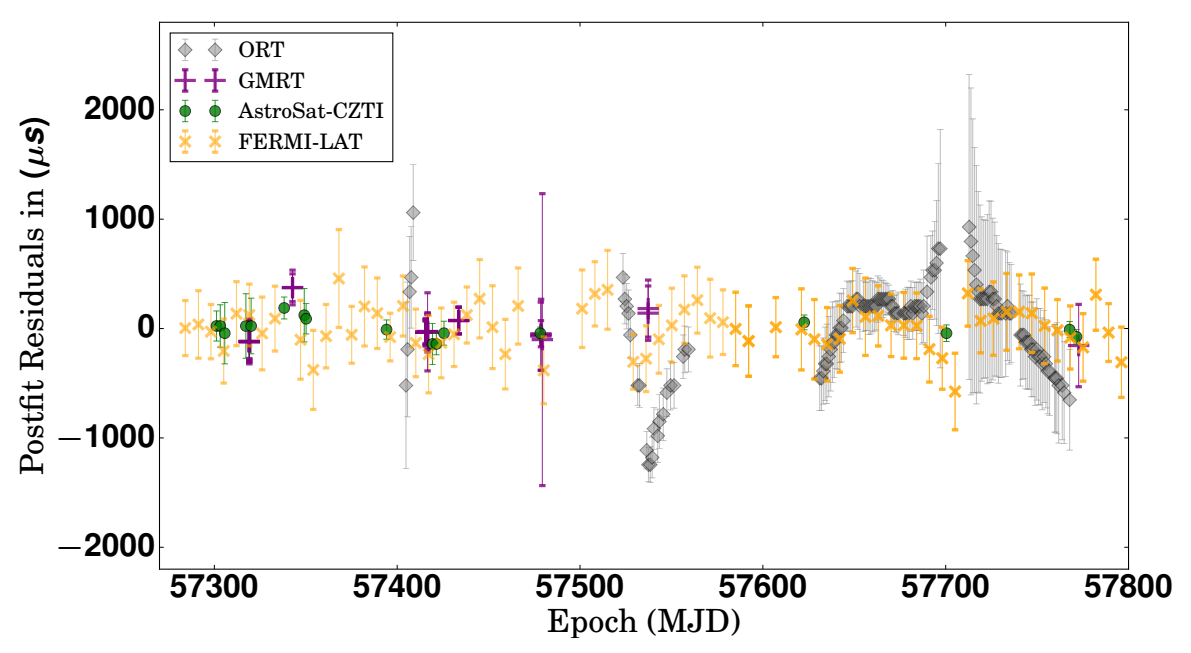

Fig. 5. Post-fit residuals after fitting the offsets between different telescopes. The symbols used are same as those used in Fig. 2.
$2.3 \mathrm{~ms}(1 \sigma)$. The relative distance between the two spacecrafts, projected in the direction of the GRB, was $877 \mathrm{~km}$ at the time of this detection, corresponding to a travel time difference of $2.9 \mathrm{~ms}$. We therefore conclude that the difference in the absolute time stamps of Fermi and AstroSat-CZTI is much less than $33 \mathrm{~ms}$ and hence no integral-period ambiguity is expected in the relative phase comparison of the Crab pulsar between these two missions.

\subsection{Timing analysis}

All the radio and high energy TOAs, analyzed using the high cadence timing solution obtained with the ORT, are shown in Fig. 2. The timing noise is clearly visible in this plot and so are the relative offsets between the telescopes. The assumed parameters of the timing model are given in Table 2 along-with the reference epochs.

The timing analysis was done using the pulsar-timing package TEMPO2. First, a reference timing solution was obtained by local fits to ORT high cadence TOAs between MJD 57282-57324 with a model involving the known astrometric and rotational parameters and DM for the pulsar. The fitted ephemeris were then used to phase connect the TOAs of all the telescopes as shown in Fig. 2. This reference ephemeris was the starting point for the subsequent analysis described below.

The main objective of this work was to estimate the offset in data acquisition pipeline of ASTROSAT. This was done by comparison of the phase of the main pulse (or TOAs) seen at the GMRT and the ASTROSAT in simultaneous observations. This is complicated by both time dependent and frequency dependent systematics in the TOAs. As is evident from Fig. 2, the pulsar shows considerable timing noise, which is independent of frequency. As the lower frequency TOAs are also affected by frequency dependent propagation effects, the timing noise was modeled using the regular cadence Fermi-LAT TOAs instead. These were fitted with a combination of eight sine waves in addition to the already fitted parameters in the reference ephemeris to model the red timing noise and obtain white timing residuals using FITWAVES model in TEMPO2 (Hobbs et al. 2006).

As the pulsar is located in a dynamic pulsar wind nebula with nebular filaments, with trapped charged particles, moving across the line of sight, the DM of the pulsar and the pulse broadening varies significantly from epoch to epoch. This introduces a systematic frequency dependent shift in barycentered TOAs, particularly significant for those derived from low radio frequency ORT topocentric TOAs. The typical variation in DM is on the order of $0.01 \mathrm{pc} \mathrm{cm}^{-3}$, which is equivalent to a shift of $21 \mu \mathrm{s}$ and $370 \mu \mathrm{s}$ at $1390 \mathrm{MHz}$ and $334.5 \mathrm{MHz}$ respectively. Thus, it is essential to correct for DM variations to obtain reliable estimates for rotational parameters and lower post-fit timing residuals. We used the constrained DMMODEL in TEMPO2 to estimate the offsets from the chosen reference DM at epochs, where simultaneous ORT and GMRT observations were available. Our measurements are plotted in Fig. 4.

The DM model was used along-with the timing noise model and the astrometric and rotational model for PSR B0531+21 for a fit to TOAs from the Fermi-LAT, the ASTROSAT-CZTI, the GMRT and the ORT. This corrects both the frequency independent and frequency dependent systematics in these TOAs allowing a more robust determination of relative offsets between the telescopes.

\section{Results and discussion}

TEMPO2 provides a way to fit the offsets between different telescopes and the resulting timing residuals are shown in Fig. 5. Rots et al. (2004) concluded that the X-ray main pulse leads its radio counterpart by about $344 \pm 40 \mu s$. We can use this measurement to find out the ASTROSAT pipeline offset. The relative offsets between the GMRT and the CZTI aboard ASTROSAT was found to be $-4716 \pm 50 \mu \mathrm{s}$. While determining these offsets was the major objective of our project, we also determined in the process the offsets between the GMRT and the ORT and the GMRT and Fermi to be $-29639 \pm 50 \mu s$ and $-5368 \pm 56 \mu s$ respectively. In addition, we verified that our timing solution fits the Jodrell Bank radio ToAs without introducing any time variable pattern.

The calibration of relative offsets between the radio and high energy emission is also important for a simultaneous radio - high energy study of GPs to look for a radio - high energy correlation. Such a study is currently underway.

Acknowledgements. This publication makes use of data from the Indian astronomy mission AstroSat, archived at the Indian Space Science Data Centre (ISSDC). The CZT Imager instrument was built by a TIFR-led consortium of institutes across India, including VSSC, ISAC, IUCAA, SAC, and PRL. The Indian Space Research Organisation funded, managed and facilitated the project. We thank the staff of the Ooty Radio Telescope and the Giant Meterwave Radio Telescope for taking observations over such a large number of epochs. Both these telescopes are operated by National Centre for Radio Astrophysics of Tata Institute of Fundamental Research. PONDER backend, used in this work, 
was built with TIFR XII plan grants 12P0714 and 12P0716. We would like to thank the anominous referee for his/her useful comments and suggestions. $A B$ would like to thank Alessandro Ridolfi for exposing to various techniques of PSRCHIVE package and Surajit Mondal for various fruitful discussions related to computational issues. We also thank Yogesh Maan for his valuable suggestions. BCJ, PKM and MAK acknowledges support for this work from DSTSERB grant EMR/2015/000515

\section{References}

Abdo, A., Ackermann, M., Ajello, M., et al. 2010, ApJS, 187, 460 Bhalerao, V., Bhattacharya, D., Vibhute, A., et al. 2017, JApA, 38, 3

Bhattacharya, D. 2017, JApA, 38, 51

Bissaldi, E., Mailyan, B., \& Meegan, C. 2017,GRB Coordinates Network, Circular Service, 1, 20543

Hankins, T., \& Eilek, J. 2007, ApJ, 670, 693

Hobbs, G., Edwards, R., \& Manchester, R. 2006, MNRAS, 369, 655

Hotan, A. W., van Straten, W., \& Manchester, R. N. 2004, PASA, 21, 302

Hu, C.-P., Ng, C.-Y., Takata, J., Shannon, R. M., \& Johnston, S. 2017, ApJ, 838,156

Hutchings, J. 2014, Ap\&SS, 354, 143

Johnston, S., \& Romani, R. W. 2004, in Young Neutron Stars and Their Environments, eds. F. Camilo, \& B. M. Gaensler, IAU Symp., 218, 315

Johnston, S., Romani, R. W., Marshall, F. E., \& Zhang, W. 2004, MNRAS, 355,31
Joshi, B. C., Kramer, M., Lyne, A. G., McLaughlin, M. A., \& Stairs, I. H. 2004 in Young Neutron Stars and Their Environments, eds. F. Camilo, \& B. M. Gaensler, IAU Symp., 218, 319

Kuiper, L., Hermsen, W., Walter, R., \& Foschini, L. 2003, A\&A, 411, L31

Lundgren, S., Cordes, J., Ulmer, M., et al. 1995, ApJ, 453, 433

Lyne, A., Pritchard, R., \& Graham Smith, F. 1993, MNRAS, 265, 1003

Mikami, R., Terasawa, T., Kisaka, S., et al. 2014, in Proc. of the 12th Asia Pacific Physics Conference (APPC12), 015106

Muslimov, A. G., \& Harding, A. K. 2003, ApJ, 588, 430

Naidu, A., Joshi, B. C., Manoharan, P. K., \& Krishnakumar, M. A. 2015, Exp. Astron., 39, 319

Ray, P. S., Kerr, M., Parent, D., et al. 2011, ApJS, 194, 17

Rots, A. H., Jahoda, K., \& Lyne, A. G. 2004, ApJ, 605, L129

Scott, D. M., Finger, M. H., \& Wilson, C. A. 2003, MNRAS, 344, 412

Sharma, V., Bhattacharya, D., Bhalerao, V., Rao, A. R., \& Vadawale, S. 2017, GRB Coordinates Network, Circular Service, 1, 20561

Singh, K. P., Tandon, S., Agrawal, P., et al. 2014, in Proc. SPIE, 9144, 91441S

Singh, K. P., Stewart, G. C., Chandra, S., et al. 2016, in Proc. SPIE, 9905, 99051E

Swarup, G., Sarma, N., Joshi, M., et al. 1971, Nat. Phys. Sci., 230, 185

Swarup, G., Ananthakrishnan, S., Kapahi, V., et al. 1991, Curr. Sci., 60, 95

Taylor, J. H. 1992, Philos. Trans. Phys. Sci. Eng., 117

Wang, J., Wang, N., Tong, H., \& Yuan, J. 2012, Ap\&SS, 340, 307

Yadav, J., Agrawal, P., Antia, H., et al. 2016, in Proc. SPIE, 9905, 99051D

Yadigaroglu, I.-A., \& Romani, R. W. 1995, ApJ, 449, 211 